\title{
Infarto agudo del miocardio asociado a uso de anorexígenos: Realidad o mito?
}

\section{Reporte de un caso}

Muñoz-Gutiérrez E* Alvarez-Cabrera MC**

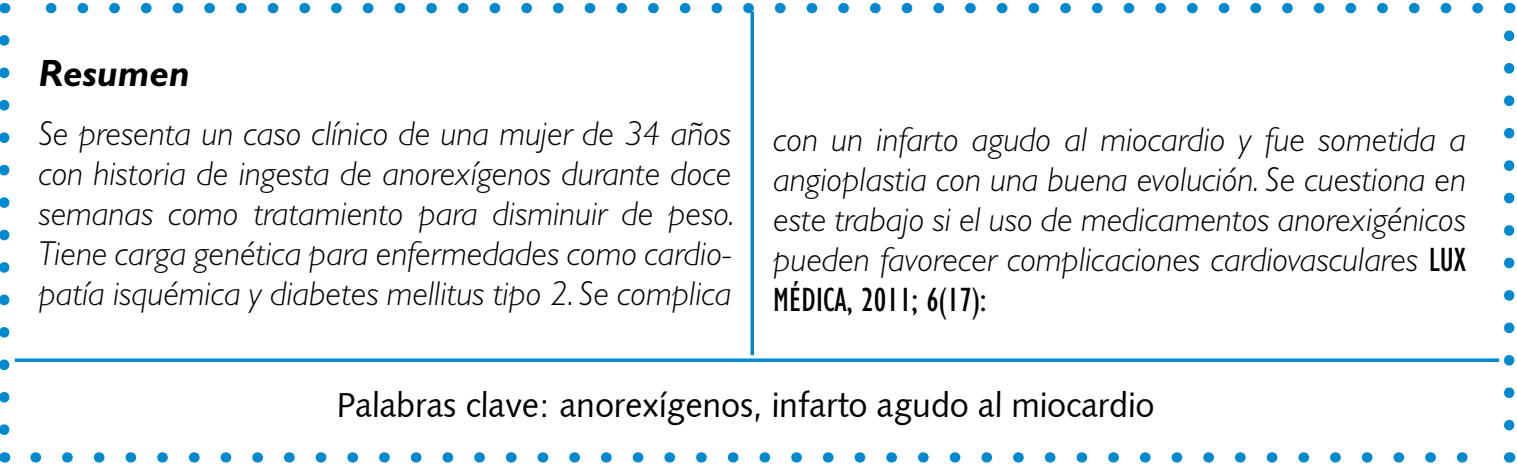

Introducción

Las principales causas de mortalidad en los jóvenes menores de 35 años, son los accidentes de tráfico relacionados con el alcohol, el suicidio y las complicaciones asociadas al síndrome de inmunodeficiencia adquirida (SIDA) ${ }^{1}$. A partir de los 35 años, la enfermedad coronaria empieza a cobrar poco a poco protagonismo y así se con-

* Cardiólogo Clínico Centenario Hospital Miguel Hidalgo.

** Estudiante del 70 semestre de la carrera de Medicina, UAA

Fecha de recibido 18 de junio 2010

Fecha de aceptación 8 de diciembre 2010

Correspondencia Dr Ellas Muñoz Gutiérrez, Cardiólogo Clínico del Centenario Hospital Miguel Hidalgo. Calle Galeana Sur \#465 Col Obraje Tel 9946720 Correo electrónico mcac_48@hotmail.com 
vierte en la primera causa de muerte en los varones mayores de 45 años y en las mujeres mayores de 65 años. A su vez, el infarto agudo de miocardio (IAM) constituye la causa mas frecuente de mortalidad isquémica del corazón, ocasionando el $68 \%$ de la mis$\mathrm{ma}^{2}$. En los últimos años esta última causa de afección cardíaca se ha incrementado por el uso cada vez mas difundido de sustancias con propiedades vasoactivas, ya sean drogas simpaticomiméticas o su utilización como coadyuvante en medicamentos para reducir de peso corporal ${ }^{3,4}$.

\section{Presentación del caso}

Paciente femenino de 32 años de edad originaria y residente de Aguascalientes, Ags; dedicada a las labores del hogar. Entre sus antecedentes heredofamiliares resalta madre con cardiopatía isquémica, hipertensión arterial sistémica y diabetes mellitus tipo 2, seis tíos maternos con diabetes mellitus tipo 2, así como abuela materna finada por cáncer de estómago. Tabaquismo de 17 años, con índice tabáquico de 7. Se define como sedentaria y cursa con dislipidemia. Tiene historia de consumo de Redotex ${ }^{R}$ y Sibutramina ${ }^{R}$ de doce meses de evolución, fármacos prescritos por un médico para disminuir el peso corporal.

Inicia su padecimiento actual el día el día 30 de abril del 2010 a las 11:30am, con dolor precordial tipo opresivo, de inicio súbito, posterior a terminar su desayuno, de intensidad inicial 3/10, aumentando progresivamente hasta 8/10, con duración mayor de 30 minutos, acompañándose de disnea, diaforesis profusa, náusea, ansiedad y parestesias de lengua. Acude en forma inmediata a médico privado. Posterior de evaluación clínica y realización de un electrocardiograma (ECG) se decide su envío al Centenario Hospital Miguel Hidalgo. Llega al servicio de urgencias a 13:20hrs. (Tiempo ventana: $1.50 \mathrm{hr}$ ).

A su ingreso a la exploración física se documentó tensión arterial de 120/70 mm $\mathrm{Hg}$, frecuencia respiratoria de 16 por minuto, frecuencia cardiaca de 97 latidos por minuto, presión arterial media de $100 \mathrm{~mm} \mathrm{Hg}$, peso $63 \mathrm{~kg}$, talla $1.64 \mathrm{~cm}$. Índice de masa corporal de 24 . Paciente activa reactiva, con facies de angustia, neurológicamente íntegra, ubicada en tiempo, espacio y persona, buen estado de hidratación y coloración de piel y tegumentos, en posición semifowler, cuello sin plétora yugular, no se auscultan soplos carotídeos, campos pulmonares sin estertores ni sibilancias. área cardiaca rítmica sin soplos, no S3, S4, sin desplazamiento de choque de punta. Abdomen blando depresible sin dolor, sin visceromegalias, peristaltismo normal, extremidades sin edema y no existe compromiso neurocirculatorio.

Se le realizó un ECG a su ingreso, que se muestra como figura 1, con imagen compatible de lesión subepicárdica anteroseptal y lateral alta. 


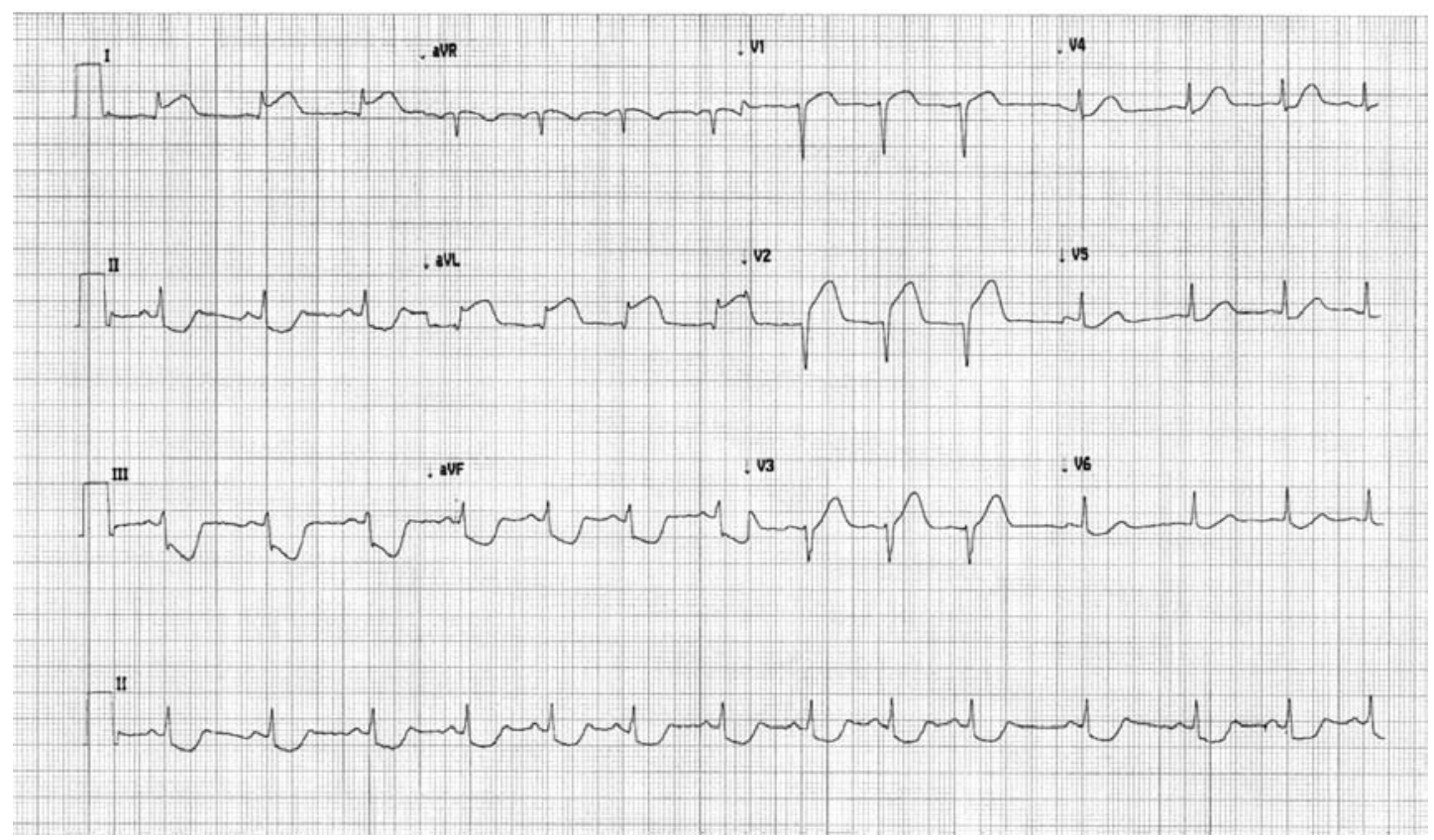

Figura 1. Imagen de lesión subepicárdica anteroseptal y lateral alta

Entre los estudios de laboratorio a su ingreso, se encuentran los siguientes:

30 abril 2010: glucosa 126 mg/dl, BUN $14 \mathrm{mg} / \mathrm{dl}$, urea $30 \mathrm{mg} / \mathrm{dl}$, creatinina 0.9 $\mathrm{mg} / \mathrm{dl}$, ácido úrico3.5 $\mathrm{mg} / \mathrm{dl}$, colesterol $155 \mathrm{mg} / \mathrm{dl}$, triglicéridos $147 \mathrm{mg} / \mathrm{dl}$, HDL $38 \mathrm{mg} / \mathrm{dl}$, fósforo $2.0 \mathrm{mg} / \mathrm{dl}$, magnesio 1.9 $\mathrm{mg} / \mathrm{dl}$, calcio $7.9 \mathrm{mg} / \mathrm{dl}$, sodio $145 \mathrm{mEq} /$ $\mathrm{L}$, potasio $4.5 \mathrm{mEq} / \mathrm{L}$, cloro $106 \mathrm{mmol} / \mathrm{L}$, enzimas cardiacas: troponina $4.48 \mathrm{U} / \mathrm{L}$, CPK MB $8.0 \mathrm{U} / \mathrm{ml}$. Tiempo de protrombina13.11, tiempo de tromboplastina parcial 24.6 segundos, fibrinógeno $365 \mathrm{mg} / \mathrm{dl}$, actividad $127 \%$ INR 0.86

Con los datos clínicos anteriores, así como los estudios de laboratorio y gabinete, se diagnostica un Infarto Agudo del Miocardio, por lo que se decide realizar angioplastía coronaria trasluminal percutánea (ACTP).

Se realizó cateterismo cardíaco documentando los siguientes hallazgos:

* Arteria Coronaria Derecha: Arteria dominante, de buen diámetro y longitud, sin lesiones aparentes, sin lesiones aparentes, flujo TIMI 3 (figura 2)

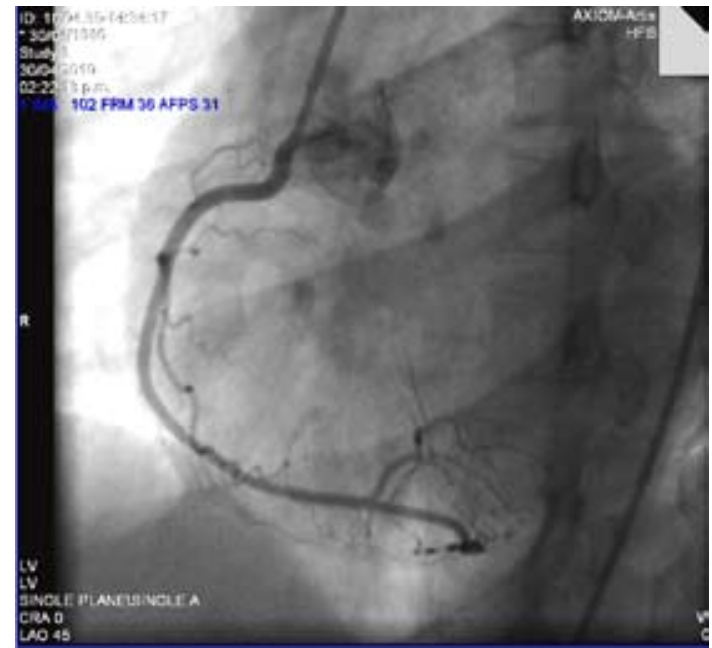

Figura 2. Arteria coronaria derecha normal sin lesiones

- Tronco de la Coronaria Izquierda sin lesiones.

- Descendente Anterior: Arteria con diámetro inferior a $2 \mathrm{~mm}$ con lesión suboclusiva en tercio proximal, diagonales con diámetro aproximado de $1 \mathrm{~mm}$, flu- 


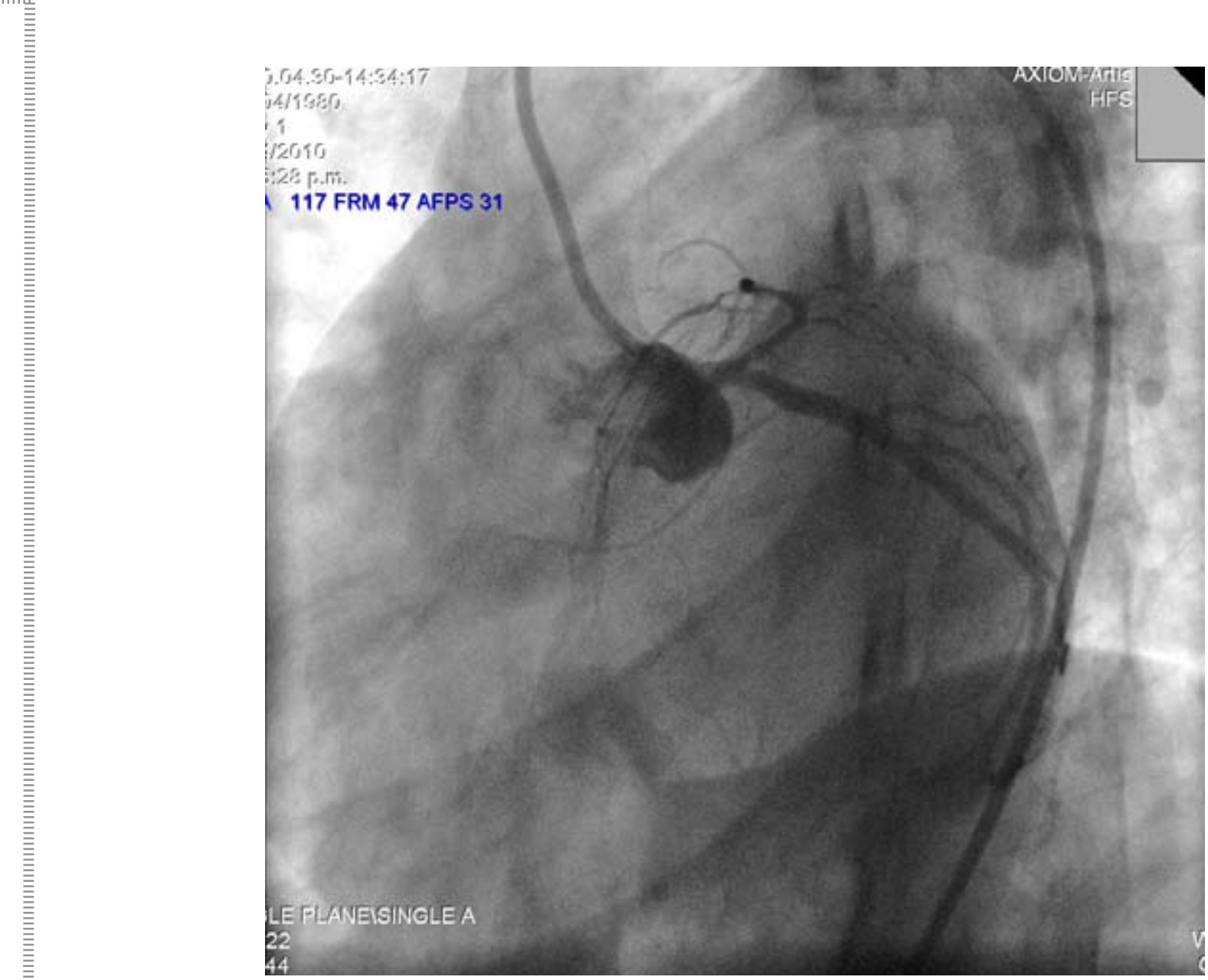

Figura 3. Angiografía coronaria en proyección oblicua anterior izquierda caudal (araña) donde se observa zona de obstrucción crítica de descendente anterior.

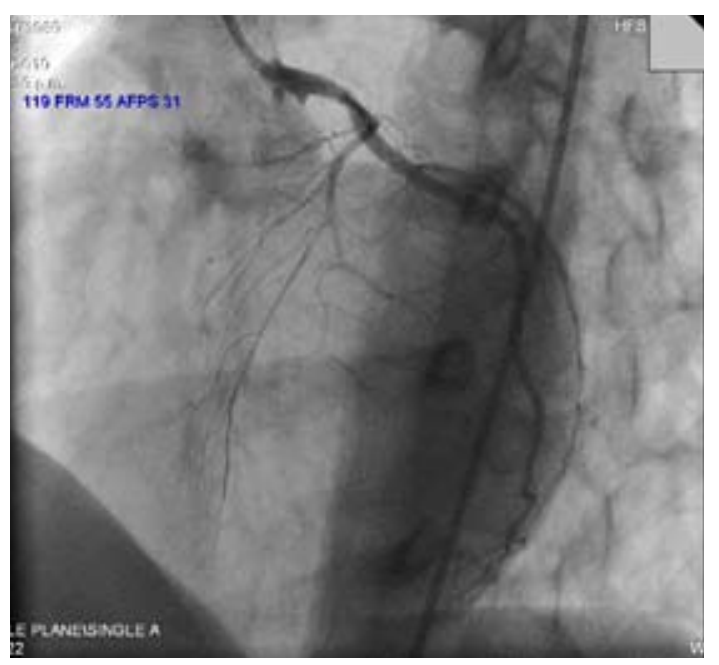

Figura 4. Angiografía coronaria en proyección oblicua anterior derecha craneal donde se evidencia la zona de estenosis y oclusión arterial.

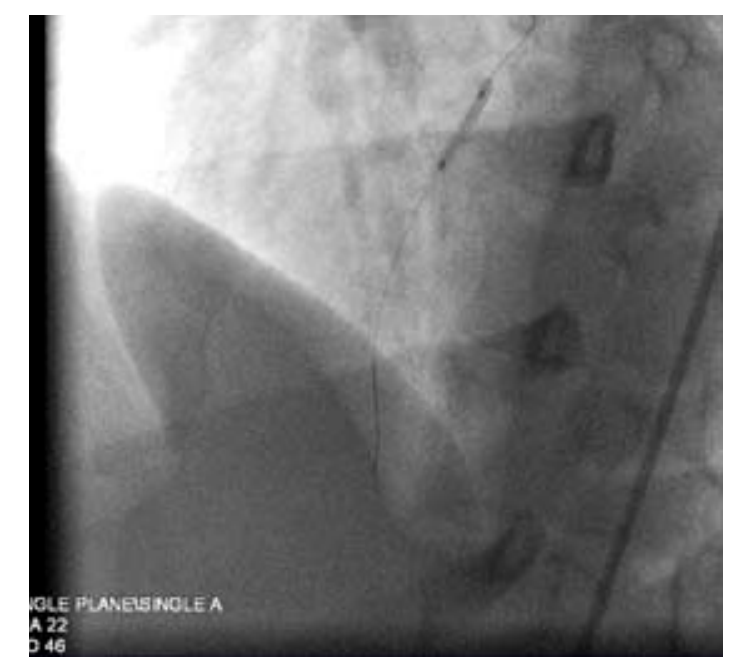

Figura 5. Balón de angioplastia. 


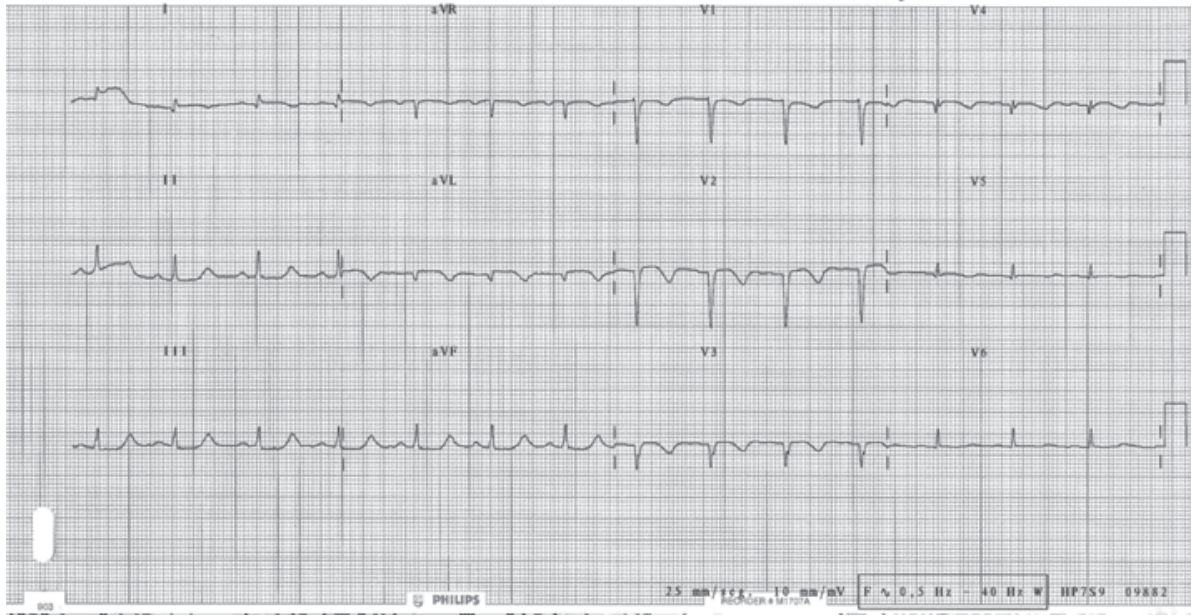

Figura 6. Imagen que muestra zona de inactividad eléctrica (necrosis) anteroseptal.

jo distal TIMI 1 (figura 3)

- Circunfleja: Arteria de buen diámetro y longitud, con imagen sugestiva de trombo en el origen de arteria, flujo TIMI 3. (figura 4)

Posterior a la angioplastia (una hora después) se le realizó otro ECG, (figura 6), con un trazo que muestra una zona de inactividad eléctrica (necrosis) anteroseptal.

Finalmente, se le realizó un ecocardiograma donde se documentan los siguientes datos:

- Ventrículo izquierdo con diámetros intraventriculares y grosor parietal dentro de rangos normales, movimiento septal paradójico, fracción de eyección del ventrículo izquierdo (FEVI) del $47 \%$ por Teichoolz y 52\% área longitud, con hipocinesia moderada septo-apical.

- Válvula mitral: área de la válvula mitral de $4.34 \mathrm{~cm}^{2}$, sin insuficiencia.

- Válvula aórtica trivalva, gradiente sistólico máximo $2.3 \mathrm{mmHg}$, sin insuficiencia.

- Válvula pulmonar con gradiente sistólico máximo $1.4 \mathrm{mmHg}$, sin insuficiencia.

- Válvula tricuspídea con insuficiencia ligera.

- Presión arterial media de la pulmonar de $19 \mathrm{mmHg}$

- No dilatación auricular

- Sin presencia de trombos o masas intracavitarias.

\section{| | | | | | | | | | | | | | | | | | | | | | | | | | | | | | | | | | | | | | | | | | | | | | | | | | | | | | | | | | | | | | | | | | | | | | | | | | | | | | | | | | | | | | | | | | | | | | | | | | ||}

- Sin derrame pericárdico.

\section{Discusión}

A pesar de que el IAM afortunadamente es una entidad poco frecuente en la población joven, constituye un importante problema debido a que conlleva un devastador efecto en la salud y un potencial alto riesgo de mortalidad. Estos pacientes jóvenes tienen además diferente respuesta a la isquemia miocárdica, presentación clínica, así como pronóstico comparado con los pacientes mayores, ya que no hay circulación colateral ni pre-acondicionamiento que hace más tolerable un evento coronario agudo como sucede en estos últimos. En la descripción de este caso se pueden observar que los antecedentes y factores de riesgo no son los característicos de la enfermedad coronaria aterosclerótica; sin embargo llama la atención un dato particular que hoy en día en nuestro medio es motivo de mitos y controversia poco fundamentados: uso de Redotex ( clorhidrato de d-norpseudofe- 
drina, triyodotironina, sulfato de atropina, aloĺna y diazepam) y Sibutramina como plan de disminución de peso y su relación con efectos cardiovasculares adversos. ${ }^{12}$ Existen en la literatura médica estudios que sugieren que el uso de Sibutramina como medicamento para combatir el sobrepeso y obesidad es seguro incluso para aquellos pacientes con factores de riesgo cardiovascular ${ }^{4,5,6,7,11}$, pero también se ha documentado en otros trabajos una asociación directa del uso de estos medicamentos con la aparición de eventos coronarios agudos en población joven del este de Europa y Asia, y en base a ello se ha prohibido su utilización en esa regiones ${ }^{8}$. Asimismo existe evidencia de casos que se han descrito en Malasia donde pacientes sin factores de riesgo cardiovascular quienes habían ingerido anorexigénicos, presentaron cambios electrocardiográficos sugestivos de IAM y en los cuales se descartaron factores de riesgo como abuso de cocaína, miocarditis viral, disec- ción aórtica, estados hipercoagulables y vasculitis autoinmunes. ${ }^{9}$ Los hallazgos de estos trabajos establecen una relación directa entre el uso de estos fármacos y alteraciones hemodinámicas por sus efectos simpáticomiméticos, aumento de tensión arterial y frecuencia cardíaca, relacionados a mayor incremento de norepinefrina y serotonina por su farmacodinámica específica sobre la inhibición de recaptura, además de una afectación directa sobre la circulación coronaria produciendo un deficiente flujo epicárdico y disfunción endotelial que en el contexto de un lecho previamente afectado por aterosclerosis puede potencialmente exacerbar la hipoperfusión e isquemia miocárdica ${ }^{10}$. Es así como hasta la fecha resultan indispensables mayores estudios que demuestren si existe una verdadera asociación entre la existencia de IAM en poblaciones jóvenes y el uso de medicamentos anorexigénicos que se continúan prescribiendo en nuestro país como lo son Redotex y Sibutramina.

\section{Bibliografía}

1 Morillas, P. Infarto agudo de miocardio en menores de 45 años. Revista española de Cardiología 2002:55 (11):1124-1131

2 Egred $M$, Viswanathan $G$, Davis K. Myocardial infarction in young adults. Postgrad Med J 2005;81:741ñ745

3 Flegal KM, Graubard BI, Williamson DF, Gail MH: Cause-specific excess deaths associated with underweight, overweight, and obesity. JAMA 2007; 298:2028-2037.

4 Birkenfeld $A L$, Schroeder $C$, Boschmann $M$, Tank J, Franke G. Paradoxical effect of sibutramine on autonomic cardiovascular regulation. Circulation 2002; 106:2459ñ2465.

5 James, W. Philip; et.al. The SCOUT study: riskbene?t pro?le of sibutramine in over-weight high risk cardiovascular patients. Eur Heart J 2005;7(Supl. L): L44ñL48.

6 Weeke, Peter; et.al. The weight lowering effect of sibutramine and its impact on serum lipids in cardiovascular high risk patients with and without type 2 diabetes mellitus - an analysis from the SCOUT leadin period. BMC Endocrine Disorders 2010, 10:3.

7 Heusser K, Tank J; et.al. In?uence of sibutramine treatment on sympathetic vasomotor tone in obese

subjects. Clin Pharmacol Ther 2006;79: 500 ñ508.

8 James, W. Philip; et.al. Effect of Sibutramine on cardiovascular outcomes in overweight and obese subjects. The New England Journal of Medicine. 2010;363:905-917

9 Azarisman S, Magdi Y, Noorfaizan S, Oteh M. Myocardical Infarction Induced by appetite suppressants in Malaysia. The New England Journal of Medicine. 2007, 357;18.

10 Heusser K, Tank J, Diedrich A, et al. Influence of sibutramine treatment on sympathetic vasomotor tone in obese subjects. Clin Pharmacol Ther 2006; 79:500-8

11 Halford JCG, Boyland EJ, Cooper SJ, et al. The effects of sibutramine on microstructure of eating behaviour and energy expenditure in obese women J. Psychopharmacol 2010;24:99-109.

12 International Obesity Taskforce prevalence data: global obesity prevalence in adults. London: The International Association for the Study of Obesity. (Accessed August 2, 2010, at http://www.iotf.org/ database/index.asp.)

13 James WPT. The SCOUT study: riskbenefit profile of sibutramine in overweight high-risk cardiovascular patients. Eur Heart J 2005; 7:Suppl:L44-L48. 